\title{
OH ZEEMAN DETERMINATION OF THE MAGNETIC FIELD AT THE GALACTIC CENTRE
}

\author{
N.E.B. Killeen, K.Y. Lo, R.J. Sault, and R.M. Crutcher \\ Astronomy Department, \\ University of Illinois, \\ 1011 West Springfield Ave., \\ Urbana, Illinois, 61801, USA.
}

\begin{abstract}
Mid and far IR measurements of polarized dust continuum emission from within the central $5 \mathrm{pc}$ of the Galaxy have been cited as evidence for magnetic alignment of dust grains. Indirect arguments have been used to infer a B-field strength of $\sim 10 \mathrm{mG}$. The presence of such high B-field would play a significant role in the hydrodynamics of the ionized and neutral gas within the region. To obtain a direct measurement of the line-of-sight B-field strength, we conducted VLA observations to try and detect the Zeeman splitting in the $1667 \mathrm{MHz} \mathrm{OH}$ absorption line against the SgrA radio source. We observed with A/B configuration, 256 $1.1 \mathrm{~km} \mathrm{~s}^{-1}$ channels, and 3 arcsec resolution. In addition, both left and right circular polarizations were acquired simultaneously. We used the maximum likelihood statistical analysis technique for low signal-to-noise ratio Zeeman data discussed by Sault, Killeen, Loushin, and Zmuidzinas (1989). Analysis of the resulting absorption line spectra is complicated by the many foreground clouds. Excluding the foreground clouds by judicious selection of spectral windows, the strongest absorption by the molecular material closest (in projection) to $\mathrm{SgrA}^{*}$ is in what Güsten et al. (1987) refer to as the redshifted cloud (it does not appear to be partaking in the general rotation of the circum-nuclear disk but is thought to be in the local vicinity of $\operatorname{Sgr}^{*}$ ). Preliminary analysis reveals no detections, and 3- $\sigma$ upper limits to the line-of-sight magnetic field throughout the red-shifted cloud of approximately $4 \mathrm{mG}$. By careful simulation of the lines (Gaussians are quite good approximations to these broad $\left[40 \mathrm{~km} \mathrm{~s}^{-1}\right]$ lines) in select spatial regions of the redshifted cloud, we find that a line-of-sight field as strong as $10 \mathrm{mG}$ should be clearly detectable at about the 10- $\sigma$ level if it is present. Our data do not show fields at this level.
\end{abstract}

\section{References}

Güsten,R. et al., 1987, Ap.J., 318, 124.

Sault, R.J., Killeen, N.E.B., Loushin, R., and Zmuidzinas, J., 1989, preprint. 